\title{
Quantitative regional curvature analysis: An application of shape determination for the assessment of segmental left ventricular function in man
}

\begin{abstract}
All traditional techniques of regional ventricular function analysis depend upon one or more assumptions about coordinate, reference, or indexing systems, ideallied ventricular geometry, and the uniformity of ventricular contraction. Therefore, a method of shape analysis was developed that allows the quantitation of regional curvature and is independent of the assumptions outlined. This was implemented on a commercial image processing unit and applied to sllhouettes of 30-degree right anterior oblique left ventriculograms. Three groups with abnormal wall motion (anterior abnormality, $n=23$; inferior abnormality, $n=23$; anterior and Inferior abnormalttes, $n=22$ ) were analyzed and compared to a group with normal regional function ( $n=22$ ). Relatively few significant quentitative curvature differences were noted at end diastole among the groups. These fow abnormalltes described a sllght increase in curvature or globularity of the anterior and inferior walls. More marked and extensive aberrations were detected at end systole. The group with anterlor wall motion disturbances showed four distinct areas of curvature abnormality. Excessive curvature was present on either side of the apex (anterior and inferoapical regions) and apical curvature was less than normal. The fourth region was in the inferior zone, which showed curvature values that were lees than normal, suggesting increased inward motion contralateral to the anterior abnormallty. The group with inferior wall motion abnormalities also showed excessive end-systolic curvature on either side of the apex (diaphragmatic and anteroapical zones) and deficient curvature at the apex. A combination of these reglonal morphologlc abnormalities was noted in the group with both anterior and inferior dysfunction. Regional shape change (end-dlastolic minus end-systolic curvature) largely reflected the same abnormallties as the analysis of end-systolic curvature alone. In conclusion, shape abnormalities secondary to regional dysfunction can be quantitated on a segmental basis. This form of analysis provides a conceptually different approach to the quantitation of regional ventricular function that is divorced from the assumptions mandated by traditional techniques. (Am Heart J 1987;113:326.)
\end{abstract}

G.B. John Mancini, M.D., F.R.C.P.(C), Scott F. DeBoe, B.S., Edward Anselmo, B.S., Sandra B. Simon, M.A., Michael T. LeFree, B.S., and

Robert A. Vogel, M.D. Ann Arbor, Mich.

Clinicians involved in day-to-day patient diagnosis and management are frequently called upon to make an assessment of regional ventricular function. Moreover, investigations designed to establish the standards of patient care are heavily dependent

From the Department of Internal Medicine, Division of Cardiology, Veterans Administration Medical Center, University of Michigan Medical School.

This study was supported in part by funds from the Veterans Administration, Washington D.C., and by the American Heart Association of Michigan, Lathrup Village, Michigan.

Received for publication March 24, 1986; accepted July 1, 1986.

Reprint requests: G.B. John Mancini, M.D., Veterans Administration Medical Center, 2215 Fuller Road, Ann Arbor, MI 48105. upon the demonstration of whether or not proposed interventions significantly alter regional function. ${ }^{1}$ It is thus important to appreciate that despite the fundamental need to recognize myocardial dysfunction, determine its extent, and monitor changes induced by therapeutic or diagnostic interventions, controversy continues as to the optimal method of measurement of this significant parameter. All traditional methods of regional function analysis in patients require assumptions about the coordinate system (measurement of motion along polar or rectangular coordinates or changes in areas outlined by polar or rectangular coordinates), the reference system (internal or external), and the indexing 

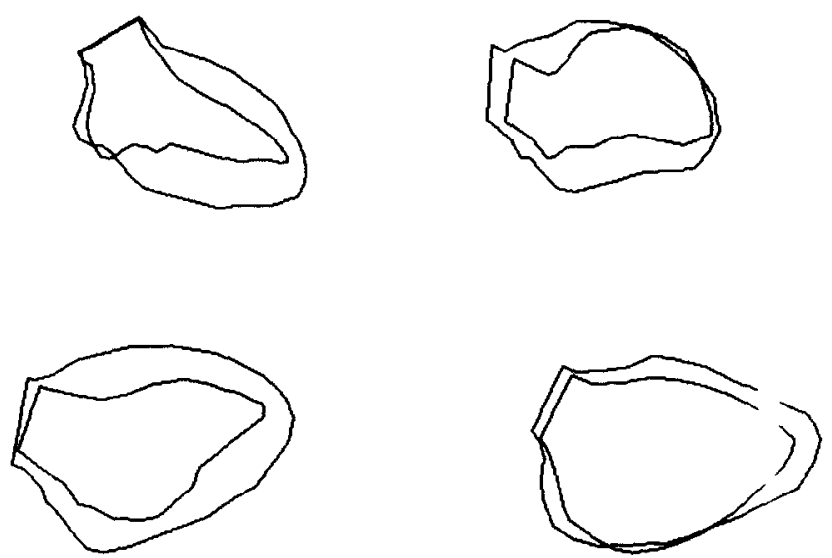

Fig. 1. End-diastolic and end-systolic outlines from patients with normal wall motion (upper left), anterior dysfunction (upper right), inferior hypokinesis (lower left), and global derangement of function (lower right). Notice that the end-systolic contours are more distinctive in shape among these examples than the end-diastolic contours. These digitized outlines were photographed from the display terminal of the image processing unit.

system (fixed or floating to account for translational and/or rotational motion). ${ }^{2}$ These, in turn, are influenced by assumptions regarding the normal geometry of the ventricle and the concentricity or uniformity of its contraction pattern. These features provide the basis for the continuing controversies in regional analysis. Despite repeated attempts to determine which combination of assumptions and approximations results in an optimal wall motion analysis system, no consensus has yet been reached. ${ }^{3-10}$

This laboratory has recently attempted to develop a conceptually different system of ventricular function analysis that abandons dependency on the many assumptions mandated by traditional techniques, is applicable to numerous imaging methods applied in man, and provides quantitative, regional information. The methodology, which measures regional shape and not wall motion per se, is based on the premise that normal and abnormal ventricles have different shapes, that aberrations of shape occur by virtue of abnormalities of contraction or relaxation, and that quantitation of shape abnormalities will reflect abnormalities of ventricular function.

Consider Fig. 1. This shows four sets of ventricular silhouettes, each representing either normal or abnormal regional function and traced with reference to stationary ribs and diaphragm (external reference system). These shapes convey to the experienced clinician the presence of normal motion

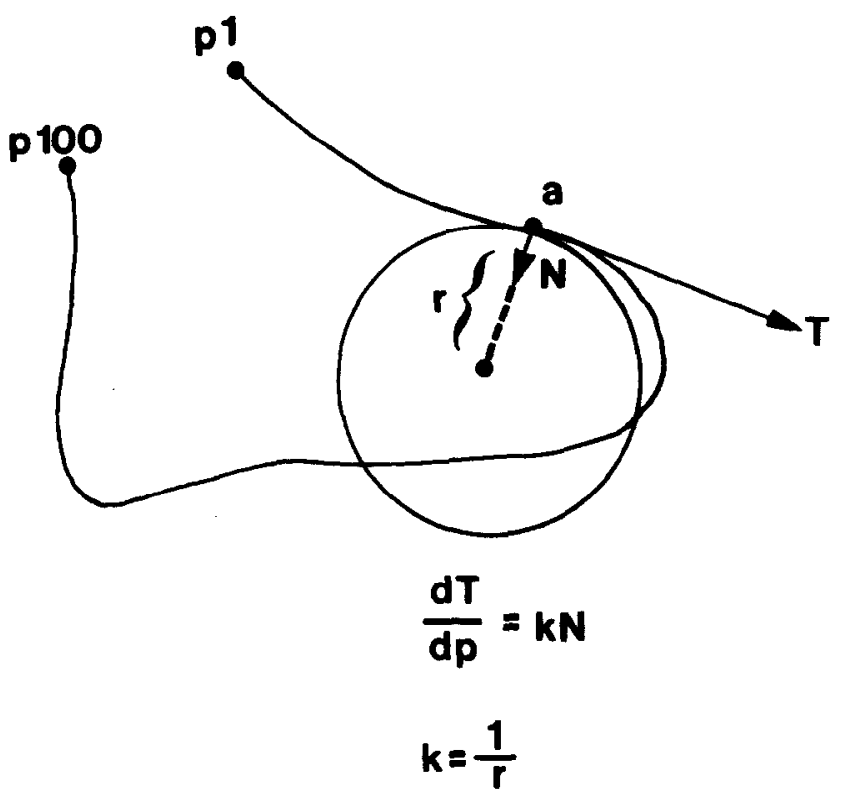

Fig. 2. The arc labelled $p$ (1 to 100$)$ is drawn to represent a ventricular silhouette. The circle with radius $r$ that intersects points $a$ tangentially is illustrated. The rate of change of the tangent vector $(T)$ with respect to changes in the arc length is equal in direction and magnitude to $k$ $N$, where $N$ is the unit vector perpendicular to $T$ and $k$ is curvature, which is equal to $1 / r$.

(upper left), abnormal inferior motion (lower left), anterior dysfunction (upper right), and global derangement of function (lower right). As no actual motion is portrayed by the stationary silhouettes, it can be argued that it is the shape information shown that allows inference as to the underlying ventricular function. Moreover, closer scrutiny suggests that it is the end-systolic outline that is most revealing about underlying normal or abnormal function. Thus, these simple observations are in keeping with the proposed hypotheses, but quantitation of these apparent shape abnormalities would be desirable. The mathematical quantity, curvature, was selected for this purpose and quantitative, regional shape characteristics of subjects with normal ventricular function were recently described. ${ }^{11}$ The purpose of this investigation was, therefore, to determine whether or not distinctive patterns of regional shape aberration could be detected and quantitated in patients with localized left ventricular dysfunction.

\section{METHODS}

Left ventriculograms obtained in the 30 -degree right anterior oblique projection were retrospectively analyzed to find patients with studies that were suitably opacified to allow accurate tracing of the endocardial outline at end diastole and end systole. Post ectopic beats were not 

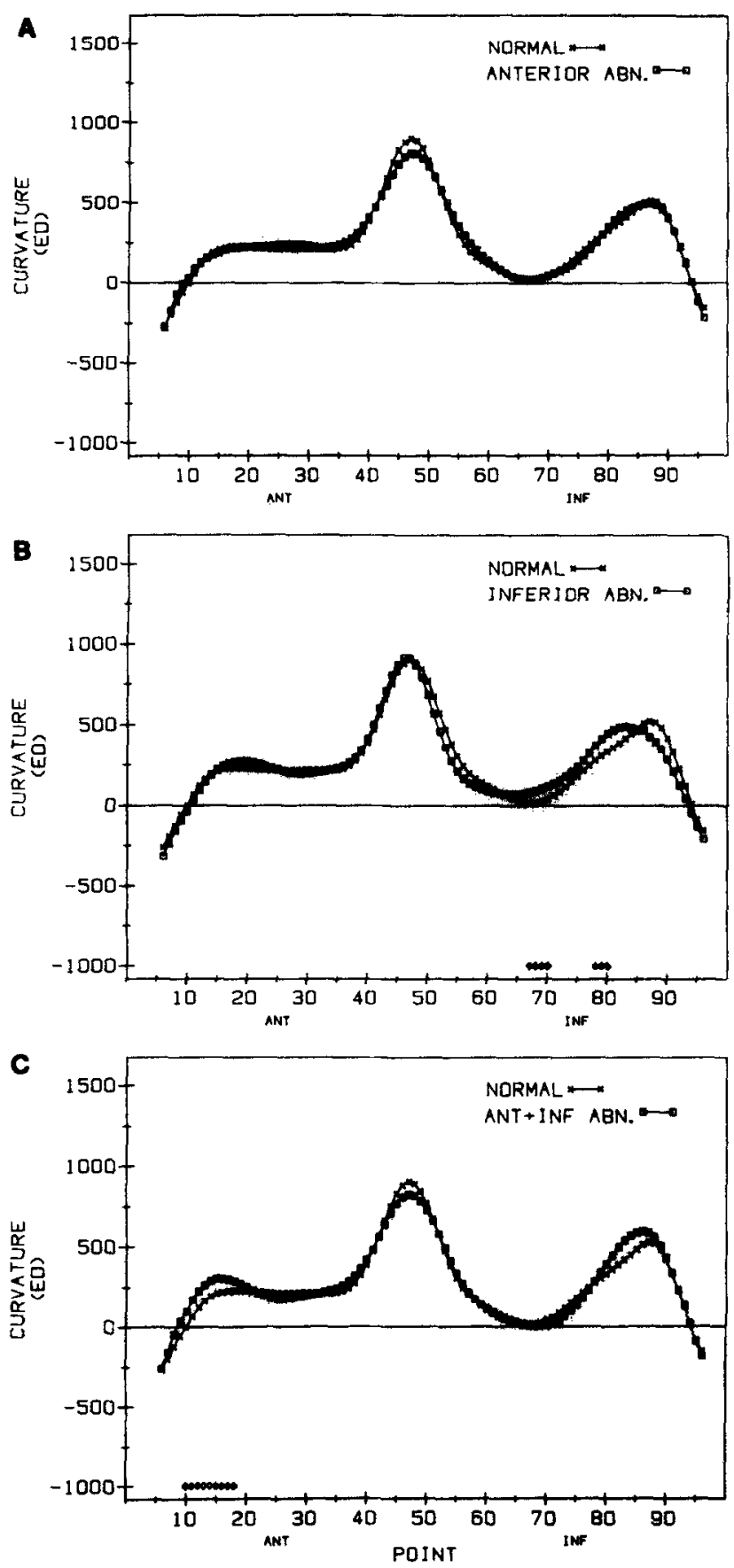

Fig. 3. Panels $A, B$ and $C$ show the end-diastolic curvature results of the anterior, inferior, and combined wall motion abnormality groups, respectively, as compared to normal results. The points on the abscissa refer to successive equidistant points on the ventricular silhouettes progressing clockwise from the anterior aortic valve plane to the junction of the mitral and aortic valves. Curvature values on the ordinate are given in dimensionless units and all graphs are drawn to the same scale. The squares show results in the abnormal subgroups and the $x$ 's show the normal mean curvature results. These are redrawn in each panel for ease of comparison. Symbols above the abscissa mark the specific points with statistically significant differences. Crosses denote $p<0.05$ and diamonds denote $p<0.01$. $A N T=$ anterior; $I N F=$ inferior; $A B N=$ abnormalities. considered for analysis, and patients with significant valvular disease, papillary muscle hypertrophy, or angiographic mitral valve prolapse were excluded. Four groups of patients were established by consensus: (1) patients with normal regional function $(\mathrm{n}=22)$; $(2)$ patients with isolated anterior wall motion abnormalities $(n=23)$; (3) patients with isolated inferior wall motion abnormalities $(n=23)$; and (4) patients with abnormalities of both the anterior and inferior regions $(n=22)$. In the absence of a true gold standard for regional wall motion, the group designations were corroborated by at least three experienced observers and also by retrospectively analyzing the catheterization reports to ensure that the original angiographer not involved in the study also gave a similar designation of the location of regional dysfunction. Twenty of the 22 subjects in the normal group constitute the study population of a prior report." Careful tracings of the endocardial outlines at end diastole and end systole were made. Papillary muscles were included within the cavity. These tracings were photographed on $35 \mathrm{~mm}$ film and projected on a Vanguard viewer that was interfaced by a video chain to a commercial digital image processing unit (No. 4100C, ADAC Laboratories, San Jose, Calif.). The tracings were digitized in a $256 \times 256$ matrix and stored.

Curvature of an arc can be described as the reciprocal of the radius of the circle that best fits a segment of the arc centered at any specified point. Consider Fig. 2. The arc $p$ (1 to 100) is drawn to represent a ventricular silhouette. $T$ is the unit vector that is tangent to the curve $p$ at point $a$. $N$ is the unit vector in a direction normal to the vector $T$. The Frenet-Serret formulas ${ }^{12-14}$ denote that $d T / d p$ (i.e., the rate of change of the tangent vector with respect to change in arc length) is equal to $k N$ where $k$ is the curvature of the arc $p(1$ to 100) at a specified point. In Fig. $2, r$, radius of curvature, is the radius of the circle that intersects point $a$ tangentially. Thus, curvature $(k)$ which is equal to $1 / r$, can be calculated by this method. Moreover, this quantitative parameter describes an intrinsic property of the shape of the arc and is independent of an external or internal reference system.

Software was developed to measure curvature, by means of the commercial image processing system as previously described. ${ }^{11} \mathrm{By}$ convention, curvature analysis for all outlines was initiated at the anterior aspect of the aortic valve plane (designated point 1 ) and proceeded clockwise in 100 equidistant steps to the junction of the mitral and aortic valve planes (designated point 100). Right-handed curves were assigned positive curvature and left-handed curves were assigned negative curvature values. Curvature, being the reciprocal of the radius of curvature, has dimensions of length ${ }^{-1}$. In order to avoid detection of curvature differences due merely to size differences and not predominantly to shape differences, all values were multiplied by the length of the enddiastolic outline. In this way, curvature could be expressed in dimensionless units, thus allowing pooling of data from ventricles of different size and comparison of normal curvature data to curvature in abnormal patients who might have enlarged ventricles.

Difficulties arise in the numerical calculation of curva- 
ture in discrete or digital curves. First, statistical noise inherent in the digitized curve is amplified by differentiation. Second, due to the approximation of a continuous curve by a discrete digital curve, numerous right-angle bends occur when positions change in row or column on a microscopic basis (quantization error). The numerical approximation for curvature makes use of the fact that the statistical noise frequency and the quantization noise frequency are significantly higher than the spatial frequency of ventricular borders, thanks to the use of $256 \times 256$ pixel digital images. In practice, quantization error of the $256 \times 256$ array is first reduced by upward scaling of the $x, y$ coordinate system by a factor of 50 , thereby providing intermediate locations for the interpolation of the curves and for more accurate division of the perimeter into 100 equally spaced segments or arc lengths. Statistical noise is also minimized by the application of a 7-point, equally weighted smoothing kernēl. Quantization error is further minimized by calculating curvature based not on adjacent points but on every fourth point, thereby ignoring the many 90-degree bends encountered when comparing immediately adjacent points. Finally, the curvature results of each point are subjected to a 3-point smoothing process so that the final value assigned to each point is an average of the value at the point in question and the values on either side of it. This methodology produces smooth, continuous curvature data from the ventricular tracings.

The software was further developed to allow accumulation of results on a point-by-point basis at end diastole and end systole so that composite curvature results for each group could be obtained. Mean and standard deviation data were calculated for all four groups, and the three abnormal groups were compared to normal results on a point-by-point basis by means of an unpaired $t$ test. Differences were considered to be significant when $p$ $<0.05$.

\section{RESULTS}

Figs. 3, 4, and 5 summarize the results and are drawn using the same scale factors. Curvature on the ordinate is given in dimensionless units. The points (abscissa) correspond to successive points on the silhouettes and progress clockwise from the anterior aspect of the aortic valve plane to the junction of the mitral and aortic valves. For ease of comparison, normal mean curvature results are duplicated in each panel. Symbols along the abscissa denote those points with curvature values significantly different from normal.

End-diastolic curvature (Fig. 3). Relatively few differences in end-diastolic curvature were noted between normal and abnormal groups. No differences were seen in patients with isolated anterior abnormalities (Fig. 3, A). In patients with isolated inferior abnormalities, curvature in the inferior wall was greater than normal (Fig. 3, B). This was significant at points 67 to 70 and 78 to 80 . This is in
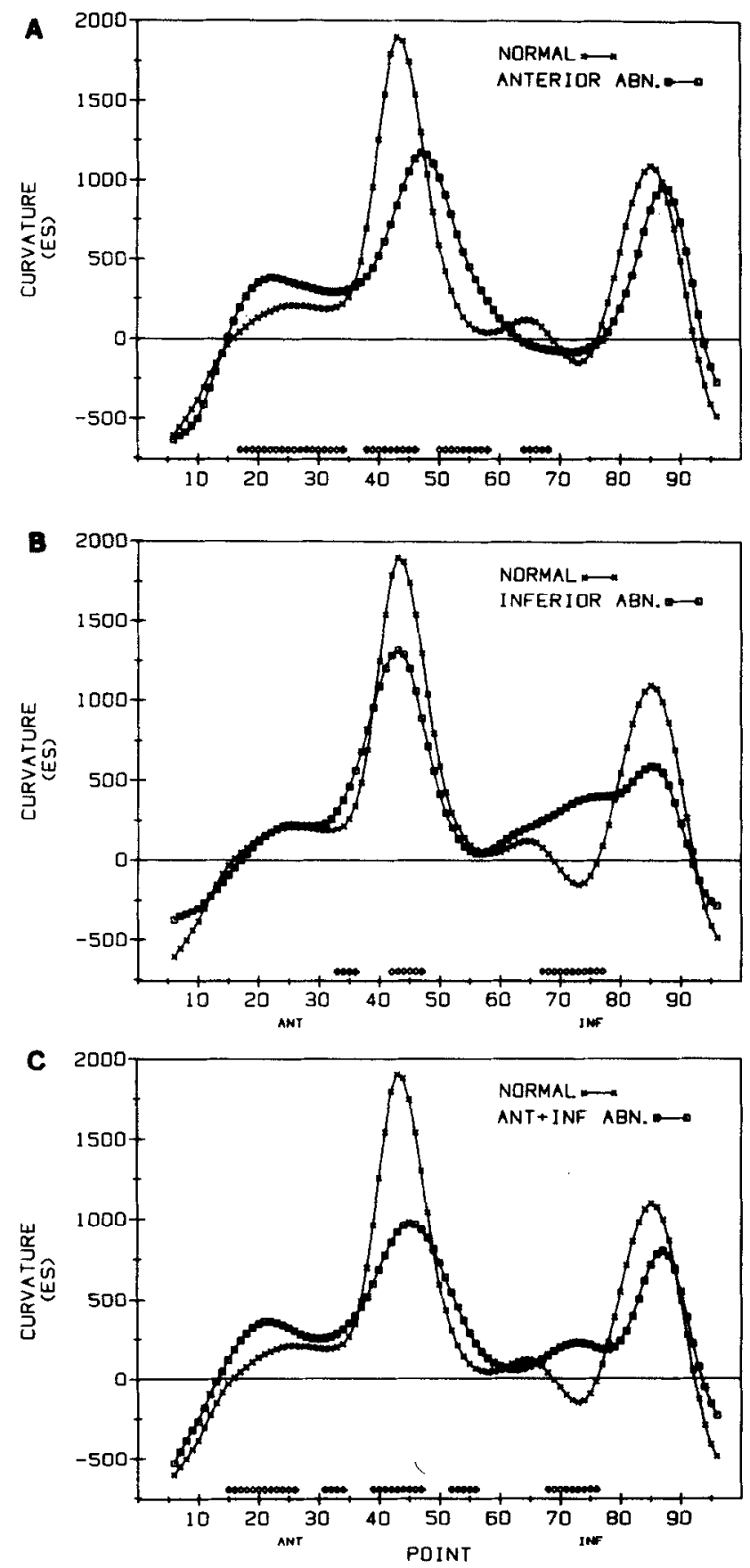

Fig. 4. End-systolic curvature results. Format and symbols are the same as in Fig. 3.

keeping with a more globular appearance of the end-diastolic inferior region in patients with regional dysfunction of this zone. Patients with combined abnormalities of regional function in the anterior and inferior walls showed a quantitative increase in curvature in only a very short zone (points 10 to 18) of the anterior region (Fig. $3, C$ ). This again is a quantitative documentation of the characteristic increase in globularity of the heart when extensive 

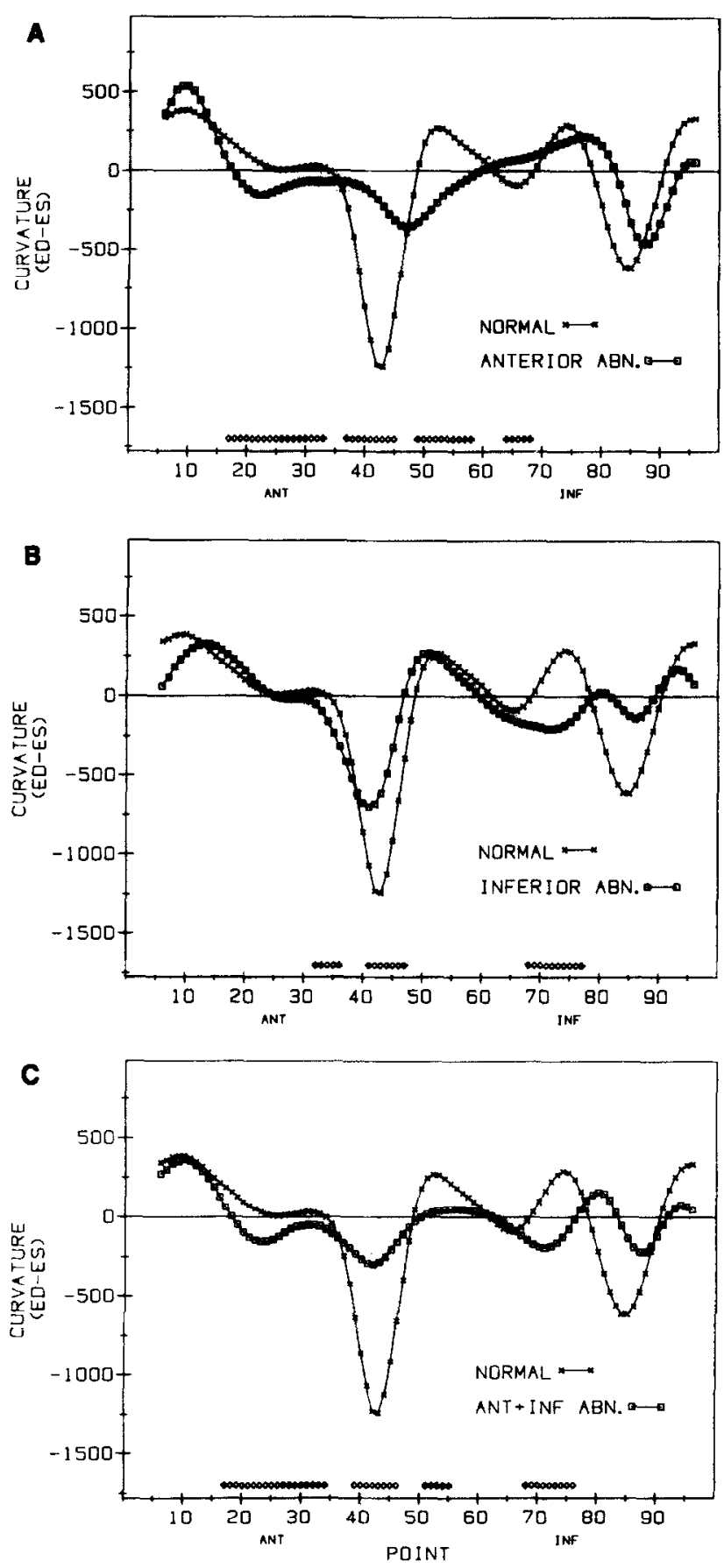

Fig. 5. Change in curvature (end-diastolic minus end-systolic). Format and symbols are the same as in Fig. 3.

dysfunction is present. It is clear, however, that shape analysis at end diastole provides relatively few diagnostic differences between normal and abnormal groups.

End-systolic curvature (Fig. 4). Analysis of endsystolic curvature demonstrated the greatest and most extensive regional shape differences among the
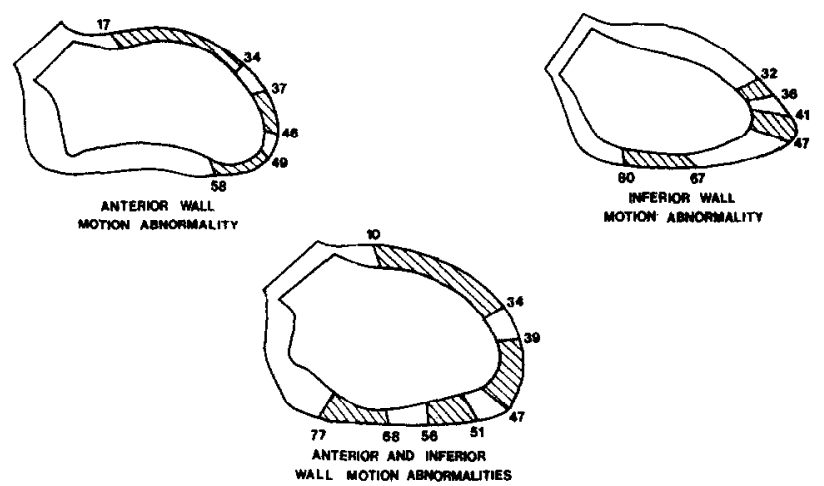

Fig. 6. Distribution of curvature abnormalities due to regional dysfunction.

groups. Patients with isolated anterior wall motion disturbances demonstrated four distinct areas of shape abnormalities. In the region spanning the anterior wall (points 17 to 34 ), curvature was excessive at end systole compared to normal results. Thus, with normal inward motion, this region has a low curvature and is therefore straighter or less globular than when inward motion is impaired. The second region of abnormality occurred between points 38 and 46 . This region corresponds to the apical zone, which characteristically has the highest curvature. Two features are evident in this zone. First, the curvature of the group with abnormal anterior function is depressed relative to controls, and second, the peak curvature in the abnormal group is shifted rightward. There are two reasons for these findings. First, anterior wall motion abnormalities frequently extend around the apex and cause it to appear more bulbous, thereby accounting for the lower curvature and rightward shift of the apical curvature peak. Second, because the analysis software divides the perimeter into 100 equally spaced segments, it is anticipated that the increased regional perimeter length due to the anterior wall motion abnormality mandates that more points will be assigned to this elongated segment than in normal patients. This effect would also tend to cause a rightward shift of the peak curvature in the presence of an anterior abnormality. The third regional abnormality noted in this group occurred at points 50 to 58 . In this zone, curvature was greater than normal, again reflecting the fact that anterior abnormalities can extend around the apex to the inferoapical region. Although the first and third regions have curvature that is greater than normal and the second (apical) region has curvature that is less than normal, taken in concert they all quantitatively connote the characteristic bulbous or globular 
nature of this region when an anterior abnormality is present. The fourth zone of significant shape difference occurred at points 64 to 68 . This region is well within the inferior zone. In contrast to the first and third regions described, this segment shows curvature values that are less than normal. Indeed, the mean curvature value in this segment is negative, suggesting a marked, inward displacement of the zone. This can be ascribed to the quantitative shape difference that occurs when contralateral hyperkinesis accompanies an anterior wall motion abnormality.

Fig. 4, panel $B$ shows the end-systolic curvature of the group with isolated inferior abnormalities compared to normal results. As with the anterior abnormality group described above, this group has several regions of distinct abnormalities. Excessive curvature (points 33 to 36 and 67 to 77) occurs on either side of the apex and deficient end-systolic curvature (points 42 to 47 ) occurs in the apical zone. Taken as a whole, these abnormalities quantitate the fact that isolated inferior abnormalities deform end-systolic ventricular shape over a substantial length that can extend beyond the apex to the anteroapical region. Second, this abnormality constitutes an increase in globularity. In contrast to the anterior wall motion group, no evidence for contralateral shape changes suggesting hyperkinesis was demonstrated. In addition, no shift in the apical curvature peak was noted. This latter phenomenon may be due to the fact that the normal inferior wall has very low and often negative curvature. A mild bulging of this zone might not significantly lengthen the perimeter and may, in fact, shorten the perimeter length. Hence, little shift in the apical curvature peak would occur.

Fig. 4, panel $C$ compares the end-systolic curvature results in the normal group and the subgroup with abnormalities of both the anterior and inferior regions. This abnormal group shows regional shape aberrations that are a combination of those in Fig. 4, panels $A$ and $B$. Hence, excessive curvature is noted in the anterior (points 15 to 26 and 31 to 34) and inferior regions (points 52 to 56 and 68 to 76), while an abnormal decrease in curvature is noted in the apical zone (points 39 to 47 ). A minimal rightward (clockwise) shift of the apical curvature peak is noted.

Change in curvature (end-diastolic minus end-systolic curvature) (Fig. 5). The comparison of the change in curvature (end-diastolic minus end-systolic curvature) is summarized in Fig. 5. In general, the location of the regional shape abnormalities in this analysis are very similar to those found in the analysis of end-systolic shape alone. Similarly, the deviations from normal are different when the apical shape abnormalities are compared to those on either side of the apex; i.e., in this comparison, shape change abnormalities involving the apex are designated above the normal curve and shape change abnormalities in the anterior and inferior regions show up below the normal curve. All of these abnormalities, however, simply reflect deficient shape changes in the abnormal patient subgroups and these, in turn, reflect the deficiency in regional shortening. In a mathematical sense, normal shape changes in the anterior region are generally of a low, positive value. This implies that end-systolic curvature is normally less than end-diastolic curvature, and this is in keeping with a flattening of the anterior wall during normal contraction. In contrast, with anterior abnormalities the anterior wall maintains an excessive degree of curvature at end-systole-i.e., it fails to "flatten" in a normal fashion. Hence, the change in curvature from end diastole to end systole is of lesser magnitude and the quantitative values occur below the normal shape change curve. Similar arguments apply to the inferior wall. In contrast, the normal apex becomes much more curved at end systole so that the difference between end-diastolic and end-systolic curvature is a very negative number. A deficient curvature increase at end systole in the presence of regional dysfunction therefore results in less negative quantitative curvature changes in the apical zone. This feature was noted in all three abnormal subgroups (Fig. 5).

Finally, it is of interest to note in Fig. $5, A$ that the region spanning points 64 to 68 shows a greater shape change than normal. This likely reflects an excessive contralateral shape change due to hyperkinesis of the inferior wall in the setting of anterior regional dysfunction, as also noted in Fig. $4, A$.

Fig. 6 provides a schematic summary of the areas demonstrating quantitative shape abnormalities.

\section{DISCUSSION}

The importance of shape characteristics of ventricular chambers has been appreciated for many centuries. ${ }^{15}$ Much of the current work, however, has been either of a qualitative nature, ${ }^{16-19}$ restricted to postmortem analyses, ${ }^{17,20-23}$ or applicable mainly to instrumented animals ${ }^{24-32}$ and patients with myocardial markers, ${ }^{33}$ or they are based on assumptions regarding coordinate systems ${ }^{34}$ and the geometry of the ventricle. ${ }^{35,36}$

$\mathrm{McDonald}^{33}$ studied the shape and movements of the human left ventricle during systole with cineangiography and also by tracking epicardial markers. 
The study focused primarily on the timing and extent of anteroposterior narrowing, descent of the ventricular base, and rotation of the apex in patients with relatively normal ventricular function. Gould et al. ${ }^{37}$ concentrated on the diameter to length ratios of the left ventricle. Vokonas et al. ${ }^{35}$ studied the dynamic geometry of the left ventricle in mitral regurgitation by determining the eccentricity of the end-diastolic and end-systolic ventricular silhouettes assuming an ellipsoid model. The results indicated a greater globularity of the ventricle in patients with mitral regurgitation, which was worse when ventricular function had decompensated. While the eccentricity measurement is appropriate for quantitating diffuse disease processes, it does not allow for regional shape assessment. Secondly, subsequent investigators have clearly shown that the shape of the normal left ventricle does not conform to the properties of an ellipse. ${ }^{19}$ This criticism also applies to the work of Dumesnil et al., ${ }^{36}$ who described a mathematical model of dynamic left ventricular geometry that was based on the assumption that the ventricle could be described by a thick-walled cylinder contracting both radially and longitudinally.

Gibson and Brown ${ }^{38}$ provided an interesting assessment of global shape change during contraction based on the relation between the volume of a solid and the nature and extent of the boundary that surrounds it. This approach was extended to ventriculographic analysis by the use of an analogous relation between area and perimeter of ventricular outlines. The change in shape index from end diastole to end systole was shown to be related to ejection fraction. Moreover, although the index was similar at end diastole in patients compared to normal subjects, end-systolic shape was clearly abnormal in patients with mitral regurgitation, congestive cardiomyopathy, mild regional wall motion abnormalities, and left ventricular aneurysms. The method assumed no idealized geometry and did not require selection of coordinate, reference, or indexing systems. However, the main drawback is that the proposed shape index is a global one and does not allow for regional ventricular assessment. A subsequent investigation by this group ${ }^{19}$ analyzed regional shape (curvature) of ventricular outlines. It was argued that since only concave surfaces are compatible with an ellipsoidal geometry, then only positive curvature values should be present for the geometric assumption to be valid. Two important points were demonstrated by the study. First, in the normal ventricle, curvature in the inferior wall was fre- quently negative (i.e., the surface is convex), and second, positive curvature in this zone, while more compatible with ellipsoidal geometry, was most often associated with regional ventricular dysfunction in patients with coronary disease. Therefore, the study demonstrated that ellipsoidal cavity geometry is not characteristic of the normal ventricle and that regional shape aberrations are related to underlying ventricular dysfunction. The study was limited, however, by the qualitative nature of shape analysis over rather broad regions of the ventricle.

The current investigation further develops the concept of shape analysis to extract information about ventricular function. The proposed method is devoid of assumptions regarding idealized geometry and does not require selection of arbitrary coordinate, reference, or indexing systems. No assumptions are made about the concentricity or uniformity of normal ventricular function, and the measurements are not invalidated by the presence of wall motion abnormalities. The technique can be extended to the characterization of three-dimensional shapes and rates of shape change. Of major importance is that the technique is quantitative and provides specific regional information. Perhaps the greatest significance of this investigation is that it provides a conceptually different approach to the assessment of regional function and this concept is applicable to many imaging techniques.

While the mathematical formulation of this method and the results in the figures may appear complex from a traditional perspective, the technique merely measures a very simple perameter, i.e., curvature, and abnormalities simply reflect regions of increased globularity, a feature that is well recognized clinically and is common to nearly all wall motion abnormalities in the setting of coronary disease. This methodology merely quantitates the sharpness of the bends in the ventricular outlines. The complexity of the analysis is, in fact, a consequence of the extremely complex morphology of the normal left ventricle. This factor is usually ignored or simplified by traditional analyses, thereby fostering the numerous assumptions outlined above.

This investigation establishes the quantitative shape abnormalities that accompany anterior and inferior wall motion disorders and determines their specific locations. It is interesting to note that the regions shown in Fig. 6 correspond closely to the regions suggested by other investigators as those that demonstrate regional dysfunction by the centerline method. ${ }^{39}$ This close agreement further supports the hypothesis that quantitative regional 
shape aberrations do indeed reflect underlying ventricular dysfunction and that such parameters can be used as the basis for alternative, assumption-free methods of assessing ventricular wall motion. It is also extremely interesting to note that contralateral hyperkinesis as documented by the centerline method may be artifactual, because that method employs an external reference system. ${ }^{6}$ The current investigation, which is based on neither an external nor internal reference system, demonstrates a contralateral shape change that can be interpreted as a reflection of contralateral hyperkinesis in the setting of anterior wall dysfunction. Therefore, this phenomenon, which clearly occurs in experimental preparations, also appears to occur in man, although the functional significance of it with respect to ventricular performance during ejection has been questioned. ${ }^{40}$

It should be emphasized that the calculations in Fig. 5 assume that the points designated at end diastole correspond to the same ventricular segments at end systole. While this may be a reasonable assumption in normal ventriculograms, ${ }^{41}$ the same is not likely to be true in the presence of wall motion abnormalities. Absolute concordance can only be ensured by implantation of radiopaque markers. The point of Fig. 5 is merely to illustrate the regional heterogeneity of shape change in both normal and abnormal ventricles. Other investigators ${ }^{34,38}$ have shown that abnormalities in parameters of shape are to be found mainly at end systole. This was anticipated and also demonstrated in this study. Moreover, the extent and location of these abnormalities at end systole were very similar to those noted when change in curvature was analyzed. Thus, the final formulation of the current curvature analysis may well entail comparison of normal and abnormal ventricles at specific phases of the cardiac cycle and not with respect to overall shape changes from end diastole to end systole, as shown in Fig. 5. If it can be shown that this approach provides the greatest accuracy in distinguishing normal from abnormal ventricles, then one can effectively overcome any assumptions regarding the comparability of ventricular segments throughout the cardiac cycle. Alternatively, consecutive assessment of shape over very short periods of time would minimize potential errors in segmental concordance during the cardiac cycle and would allow measurement of continuous shape changes on a regional basis. This could be reasonably achieved with frame-by-frame analysis of cineventriculograms and would allow determination of shape abnormalities throughout systole and diastole. This possibility requires further investigation to determine whether the approach is superior to detection of shape abnormalities at end systole alone.

A second important issue relates to the wellknown limitations of angiocardiography in defining the endocardial surface of the ventricular chamber due to papillary muscles and trabecular infolding. ${ }^{2}$ The usual methods of minimizing this problem were employed. Care was taken to include all visible contrast and papillary muscles within the ventricular outlines. This is a general limitation that affects all quantitative techniques applied to cineangiograms. Nevertheless, the concept proposed in this study can be applied to other imaging methods, such as echocardiography or cine-computed tomography, that may more accurately delineate endocardial surfaces.

A third important consideration is that for comparability from patient to patient the method does require the designation of uniform starting and ending points for the curvature calculations. The points selected for this purpose were the anterior aspect of the aortic valve and the junction of the mitral and aortic valves, respectively. These points are easily identified in cineventriculograms and are not expected to be influenced by regional wall motion abnormalities. These starting and ending points have been successfully used by other investigators to overcome potential indexing problems that occur when less constant morphologic features, such as those of the apex in the presence of wall motion disorders, are used.$^{39}$ Application of the technique to other imaging methods will require similar decisions as to where to begin and end the curvature calculations. Depending on the imaging technique and views, papillary muscles may also provide suitable alternatives to valve planes. Applications in computed tomography and magnetic resonance imaging of the heart would allow extension of these concepts to characterization of shape in three dimensions.

In conclusion, this study describes a conceptually new approach to the assessment of ventricular function that is applicable in man, can be performed on commercially available image processing systems, may be useful in several imaging methodologies, and abandons numerous assumptions mandated by traditional methods. The study demonstrates that normal shape can be quantitatied on a regional basis and aberrations caused by localized dysfunction can be detected. Furthermore, the study delineates the specific shape abnormalities and their locations. These encouraging initial results provide impetus 
for further studies analyzing the detailed relationship between quantitative shape parameters and regional function as well as a determination of the sensitivity and specificity of this method compared to traditional wall motion analysis measurements. Shape changes in the different phases of ejection and filling will also be important to establish.

\section{REFERENCES}

1. Braunwald E. The aggressive treatment of acute myocardial infarction. Circulation 1985;71:1087.

2. Ingels NB, Daughters GT, Stinson EB, Alderman EL. Evaluation of methods for quantitating left ventricular segmental wall motion in man using myocardial markers as a standard. Circulation 1980;61:966.

3. Gelberg JH, Brundage BH, Glantz Z, Parmley WW. Quantitative left ventricular wall motion analysis: Comparison of area chord and radial methods. Circulation 1979;59:991.

4. Karsch KR, Lamm U, Blanke H, Rentrop KP. Comparison of nineteen quantitative methods for assessment of localized left ventricular wall motion abnormalities. Clin Cardiol 1980;3:123.

5. Chaitman BR, Bristow JD, Rahimtoola SH. Left ventricular wall motion assessed by using fixed external reference systems. Circulation 1973;48:1043.

6. Clayton PD, Jeppson GM, Klausner SC. Should a fixed external reference system be used to analyze left ventricular wall motion. Circulation 1982;5:1518.

7. Sigel H, Nechwatal W, Kress P, Stauch M. Interobserver and intermethod variation in evaluation of regional wall motion of the left ventricle. Cardiovasc Intervent Radiol 1983;6:14.

8. Ingels HB Jr, Mead CW, Daughters GT, Stinson EB, Alderman EL. A new method for assessment of left ventricular wall motion. Comput Cardiol 1978; 57.

9. Goodyer AVN, Langou RA. The multicentric character of normal left ventricular wall motion. Implications for the evaluation of regional wall motion abnormalities by contrast angiography. Cathet Cardiovasc Diagn 1982;8:225.

10. Sheehan FH, Bolson EL, Dodge HT, Mitten S. Centerline method-comparison with other methods for measuring regional left ventricular motion. In: Sigwart U, Heintzen PH, editors: Ventricular wall motion. New York: Thieme-Stratton, Inc., 1984: 139.

11. Mancini GBJ, LeFree MT, Vogel RA. Curvature analysis of normal ventriculograms: Fundamental framework for the assessment of shape changes in man. Comput Cardiol, $1985 ; 141$.

12. Spiegel MR. Vector analysis. New York: McGraw-Hill Book Company, Inc, 1959: 35.

13. Pavlidis $\mathrm{T}$. Algorithms for graphics and image processing. Rockville, Md.: Computer Science Press, 1982: 160.

14. Beyer WH. CRC standard mathematical tables. Boca Raton, Fla: CRC Press, Inc, 1981: 358.

15. Willis R, William Harvey: A history of the discovery of the circulation of the blood. London: Kegan Paul \& Co, 1878: 185.

16. Rushmer RF, Crystal DK. Changes in configuration of the ventricular champers during the cardiac cycle. Circulation 1951;4:211.

17. Grant RP. Architectonics of the heart. AM HEART J $1953 ; 46: 405$.

18. Burton AC. The importan if the shape and size of the heart. AM HEART J 1957;54:

19. Greenbaum RA, Gibson DG. Regional non-uniformity of left ventricular wall motion in man. Br Heart $J$ 1981;45:29.

20. Woods RH. A few applications of a physical theorem to membranes in the human body in a state of tension. J Anat Physiol 1982;26:362.
21. Ross J, Sonnenblick EH, Covell JW, Kaiser GA, Spiro D. The architecture of the heart in systole and diastole. Technique of rapid fixation and analysis of left ventricular geometry. Circ Res 1967;21:409.

22. Streeter DD, Hanna WT. Engineering mechanics for successive states in canine left ventricular myocardium. I. Cavity and wall geometry. Circ Res 1973;33:639.

23. Hutchins GM, Bulkley BH, Moore GW, Piasio MA, Lohr FT. Shape of the human cardiac ventricles. Am J Cardiol 1978;41:646.

24. Hawthorne EW. Instantaneous dimensional changes of the left ventricle in dogs. Circ Res 1961;9:110.

25. Bove AA. Radiographic evaluation of dynamic geometry of the left ventricle. J Appl Physiol 1971;31:227.

26. Stool EW, Mullins CB, Leshin SJ, Mitchell JH. Dimensional changes of the left ventricle during acute pulmonary arterial hypertension in dogs. Am J Cardiol 1974;33:868.

27. Bemis CE, Serur JR, Borkenhagen D, Sonnenblick EH, Urschel $\mathrm{CW}$. Influence of right ventricular filling pressure on left ventricular pressure and dimension. Circ Res 1974; 34:498.

28. Rankin JS, McHale PA, Arentzen CE, Ling D, Greenfield JC, Anderson RW. The three-dimensional dynamic geometry of the left ventricle in conscious dogs. Circ Res 1976;39:304.

29. Badke FR. Left ventricular dimensions and function during right ventricular pressure overload. Am J Physiol 1982; 242:H611.

30. Visner MS, Arentzen CE, O'Connor MJ, Larson EV, Anderson RW. Alterations in left ventricular three-dimensional dynamic geometry and systolic function during acute right ventricular hypertension in the conscious dog. Circulation 1983;67:353.

31. Slutsky RA, Peck WW, Mancini GBJ. The effects of pulmonic and aortic constriction on regional left ventricular thickening dynamics, geometry and the radius of septal curvature. Analysis by gated computed transmission tomography. Invest Radiol 1974;19:374.

32. Bookstein FL. A geometric foundation for the study of left ventricular motion: Some tensor considerations. In: Buda AJ, Delp EJ, editors: Digital cardiac imaging. Boston: Martinus Nijhoff Publishers, 1985: 65.

33. McDonald IG, The shape and movements of the human left ventricle during systole: A study by cineangiography and by cineradiography of epicardial markers. Am J Cardiol 1970; 26:221.

34. Brower FW, Meester GT. Quantitative cardiomorphology of the left ventricle: A new approach to the measurement of regional wall motion. Comput Cardiol 1981; 67 .

35. Vokonas PS, Gorlin R, Cohn PF, Herman MV, Sonnenblick EH. Dynamic geometry of the left ventricle in mitral regurgitation. Circulation 1973;48:786.

36. Dumesnil JG, Shoucri RM, Laurenceau J-L, Turcot J. A mathematical model of the dynamic geometry of the intact left ventricle and its application to clinical data. Circulation 1979;59:1024.

37. Gould KL, Lipscomb K, Hamilton GW, Kennedy JW. Relation of left ventricular shape, function and wall stress in man. Am J Cardiol 1974;34:627.

38. Gibson DG, Brown DJ. Continuous assessment of left ventricular shape in man. Br Heart J 1985;37:904.

39. Bolson EL, Kilman S, Sheehan FH, Dodge H'l. Left ventricular segmental wall motion: A new method using local direction information. Comput Cardiol 1980; 245.

40. Lew WYW, Chen Z, Guth B, Covell JW. Mechanisms of augmented segment shortening in nonischemic areas during acute ischemia of the canine left ventricle. Circ Res 1985;56:351.

41. Doss JK, Hillis LD, Curry G, Lewis SE, Dehmer GJ, Parkey RW, Mitchell JH, Willerson JT. A new model for the assessment of regional ventricular wall motion. Radiology $1982 ; 143: 763$. 